\title{
The influence of surface coatings on the differences between numerical and experimental results for samples subject to a pulse thermography examination
}

\author{
by M. Susa*/** X. Maldague*, S. Svaic ${ }^{* *}$, I. Boras ${ }^{* *}$ and A. Bendada* \\ *Laval University - Département de génie électrique et informatique, Québec, Canada \\ ${ }^{* *}$ Faculty of Mechanical Engineering and Naval Architecture, Zagreb, Croatia
}

Key words: NDT, Pulse thermography, Surface painting, Numerical modeling, Thermal contrast

\begin{abstract}
This paper presents the analysis of the influence that black surface paint layers have on the differences in the results obtained from numerical modelling and those obtained experimentally. Surface paints are commonly used for the purposes of pulse experiments in order to increase the sample surface emissivity and help enhance the signal obtained. The paper argues that it is important to include these paint layers in the numerical analysis either directly, as additional material layer, or alternatively, to estimate their influence and take it into account when comparing the corresponding results.
\end{abstract}

\section{Introduction}

Infrared thermography is a method of non-contact surface temperature measurement. The measurement principle is based on the radiation law, which puts into relation the energy radiated from the object's surface and its surface temperature. Two different surfaces do not necessarily radiate the same amount of energy when they are at the same temperature. The amount of energy emitted depends also on the object surface emissivity, a coefficient ranging from 0 to 1 and putting into relation the energy emitted from the real object surface at a given temperature and the energy that the ideal blackbody surface would emit at the same temperature. The higher the surface emissivity, the closer the real object surface to the ideal blackbody surface and the higher the quantity of energy emitted at a given temperature.

In non-destructive testing, more often than not, surface characteristics of materials that are subject to pulse thermography (or other IR thermography) testing procedures, have poor surface emissivity properties. In the case of different metals, such as aluminium and steel, emissivity values range from 0.1 to 0.4 [1]. Knowing already that the thermal signal has a relatively low signal to noise ratio (SNR), especially when the temperatures obtained are not much higher with respect to room temperature, different strategies of signal enhancement are commonly used [2]. High emissivity surface paints $(\varepsilon>0.95)$ are applied on tested sample surfaces prior to experiment in order to increase the signal that is emitted from the sample surface and captured by the IR camera. Those layers of paint are often neglected when thermal contrast analysis is made, assuming therefore that their influence on the experimental results is negligible. An experiment, in which a metal flat-bottom hole sample plate was tested, revealed large differences when the results were compared between the black-painted region and a small region where the surface black paint fell off over the time. This observation encouraged further research which was directed towards a Plexiglass model. It was expected that Plexiglass was to be easier to work with due to its lower conductivity and, therefore, to the slower rate at which the changes in the sample appear during the experiment, thus making it easier to see the differences. As a result of that research, this article demonstrates to what extent the surface paints can influence the maximum thermal contrast as well as the time of its appearance and why they should not be neglected completely when numerical models are compared to the corresponding experimental sample.

\section{Relevant literature overview}

In an attempt to develop a method that would enable the quantitative subsurface defect characterisation based upon the maximum thermal contrast, many authors have used the theoretical models of heat transfer applied to the given sample tested via pulse thermography inspection procedure. In such a way, they were able to determine the theoretical thermal contrasts which then could be compared to those obtained by experiments for corresponding defects. Different approaches were adopted depending on the model assumptions with respect to the model geometry (1D, 2D or 3D), to the heat transfer mechanisms included, as well as to the mathematical method used to obtain the solution of the problem previously defined. Some models included development of analytical models assuming the sample to be a semi-infinite body subject to a short Dirac pulse of high intensity and with boundary conditions defined to be adiabatic, so that no heat was exchanged between the object and its environment after the heat pulse was completed. $[3,4,5]$ Others treated the models of delaminations between the two material layers and used integral methods (Fourier, Laplace ...) in order to obtain the solution of the inverse problem in the explicit form in the transformed time-space domain. [6, 7, 8, 9] Application of the finite difference method as well as the finite element method was also reported in several articles, treating the problem of heat transfer in tested samples. It was found useful to turn to these mathematical tools especially in cases where modelling of complex samples was needed and in those cases where boundary conditions included heat transfer by radiation and where some properties of the materials used in modelling were temperature dependant $[10,11$, 
$12,13,14,15]$. In most cases, defect depth retrieval was the main interest of the characterization, but in some cases defect size and also defect thermal property determination through the defect thermal resistance evaluation were described. Both reflection mode and transmission mode experiments were considered, in some cases simultaneously for both defect depth retrieval, as well as the defect thermal resistance evaluation [7].

However, results obtained in cases where they were compared with actually measured experimental data, showed significant discrepancies with respect to those obtained numerically. Among reasons given as a possible explication for these differences, authors mention uncertainty with respect to material properties used in modelling, neglecting of some heat transfer mechanisms, measurement errors and other reasons. However, none of the studies includes the analysis of the influence that surface paint used for emissivity enhancement might have on the model results. Evaluation of this impact for the case of Plexiglass flat-bottom plate samples is the subject of this publication.

\section{Experiment and sample model description}

\section{Sample and experimental set-up}

A flat-bottom hole sample made of Plexiglass with 6 holes all of the same diameter and located at different depths was used in experiment. Figure 1 shows the geometry of the tested sample. The plate surface that was to be exposed to flash heating was painted with black paint of high emissivity $(\varepsilon>0.96)$. The experiment was conducted in reflection mode. Two high power $(6.4 \mathrm{~kJ})$, low duration Balcar FX 60 flash lamps were used as excitation sources. The pulse duration was $10 \mathrm{~ms}$. Acquisitions were made at a frequency of $12.46 \mathrm{fps}$, so that with a maximum number of images acquired a sufficient time duration of the experiment could be captured. An infrared 14 bits ThermaCAM TM Phoenix® camera from FLIR Systems operating in the 3-5 $\mu$ m range was used (InSb 640x512 FPA, Stirling closed cycle cooler).

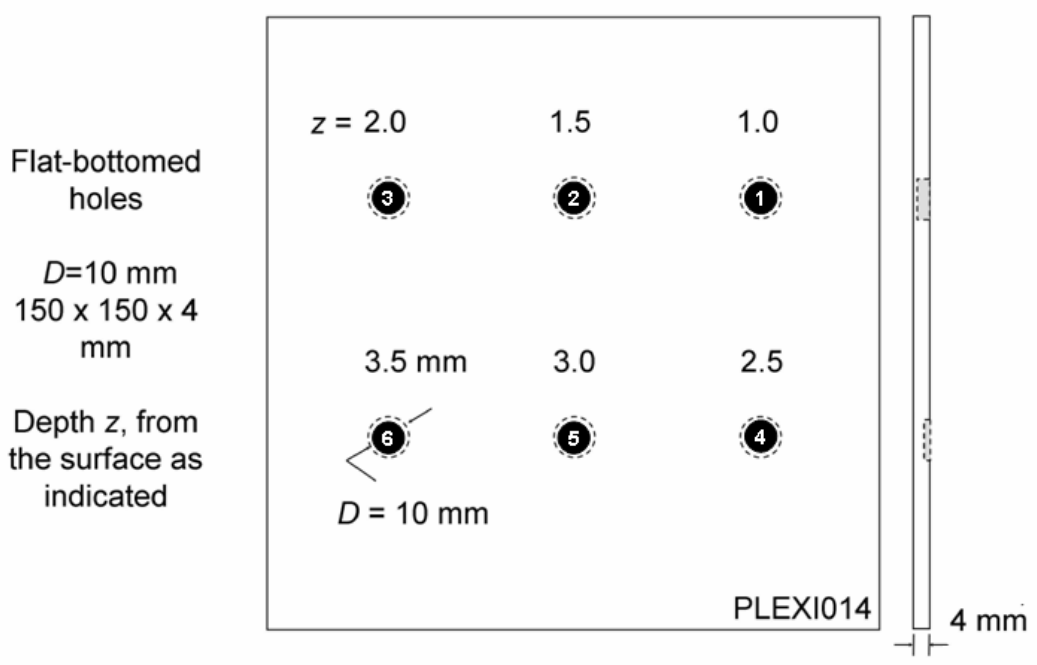

Fig. 1. Tested sample geometry

\section{Numerical model}

The physical model assumed 3D heat transfer conditions within a homogeneous Plexiglass plate (density $\rho=1190 \mathrm{~kg} / \mathrm{m}^{3}$, thermal conductivity $\mathrm{k}=0.19 \mathrm{~W} /(\mathrm{mK})$, specific heat capacity $\left.\mathrm{c}_{\mathrm{p}}=1470 \mathrm{~J} /(\mathrm{kgK})\right)$ of a geometry corresponding to the sample specifications. Boundary conditions included heat transfer by convection and radiation on all sample boundaries with variations on the surface emissivity properties as well as on the heat transfer coefficients by convection corresponding to the assumed experimental conditions. Two different cases were analyzed. In the first model no paint layer was included, whereas the second model had a $25 \mu \mathrm{m}$ thick surface paint layer. Since neither the precise thickness nor the exact thermal properties of paint were known, the thickness assumed was within the average thickness of such paint layers as reported in literature, while three different simulations were made in order to obtain and compare the differences in the results for three paints (Paint I, Paint II and Paint III - details discussed below) of different thermal properties, as specified in Table 1.

At the same time, the numerical model of the sample plate with the corresponding physical model conditions was developed and simulations of pulse experiment were done. The software COMSOL 3.2 from Comsol, Inc was used for numerical simulations. The software problem solution is based on the finite element method, providing a powerful tool for complex physical problem analysis, particularly when the geometry of the model is of a more complex nature and when non-linear problems are treated. The time stepping used was variable and adjusted so that rapid changes in the heating up period as well as in the first part of the cooling down process could be adequately treated, permitting at the same time to reduce the overall number of time steps as larger time-steps were applied towards the end of the period modelled. In this particular case an additional 
difficulty was to achieve a model sufficiently sophisticated to take into account large temperature differences close to the sample surface right after the heat pulse application. Taking into account the software limitations, this was particularly sensitive when a model with a thick paint layer was analysed. The large disproportion between the paint layer thickness and the other two model dimensions made it impossible to simulate the whole model at the same time with sufficient accuracy and within the limitations of reasonable time and computer power consumption. Therefore a choice to simulate one defect at a time in the case of the model with a paint layer was made. The geometry and the mesh used in simulations for both cases are shown in Figure 2. Figure 2.b shows the model of the shallowest of 6 defects, the one that is located $1 \mathrm{~mm}$ beneath the sample surface. In all, six different models for each of the 6 defects located at different depths were made for simulations including the surface paint layer.

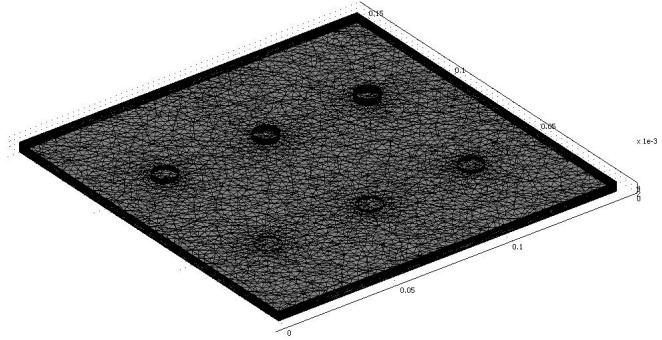

a)

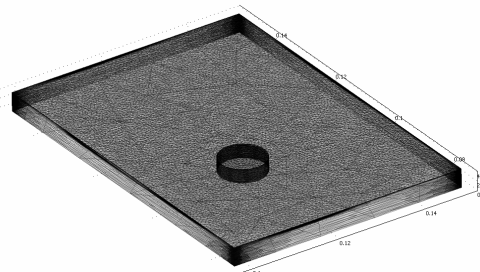

b)

Fig. 2. Geometry and mesh of models used in two simulations

The main concern was whether the results of the model including the whole sample (integral model) will be comparable to those of the segment model. In order to verify this assumption, a simulation of one segment was made without a paint layer so that the two model results can be compared for both sane and defective areas. The case of the shallowest defect was chosen since the thermal contrast was expected to be the largest in that case and any important differences in two model results would be more easily noticed. The difference in maximum thermal contrast between the results of the two models is within the limits of the IR camera sensitivity $\left(0.02^{\circ} \mathrm{C}\right)$ and represents less than $0.2 \%$ of the maximum contrast value obtained. Therefore, it was concluded that differences in results of the two models are so small that they can be completely neglected and the results of the two models can be taken as comparable.

Finally, it should be mentioned that the initial condition used in the model, the overall sample temperature before heating, was taken to be equal to the room temperature as measured prior to the experiment, since the sample was subjected to the given room temperature conditions for a sufficient time period to attain a thermodynamical equilibrium with the environment. Sample surface temperature measurement was also used for determination of its emissivity, which was possible since the camera was calibrated using a standard blackbody, right after the experiment was performed. Other model parameters used in simulations and that were not a priori known, such as heat source density and heat transfer coefficients on the sample surfaces, were adjusted in order to fit the experimental data. The criteria used for the parameters determination was the equal behaviour of the sane area regions as obtained from experiment and from simulations.

\section{Main results and discussion}

Figures $3 a$ and $3 b$ show the surface temperature distributions of the Plexiglas plate 30 seconds after the strong heat pulse of very short duration has been applied.

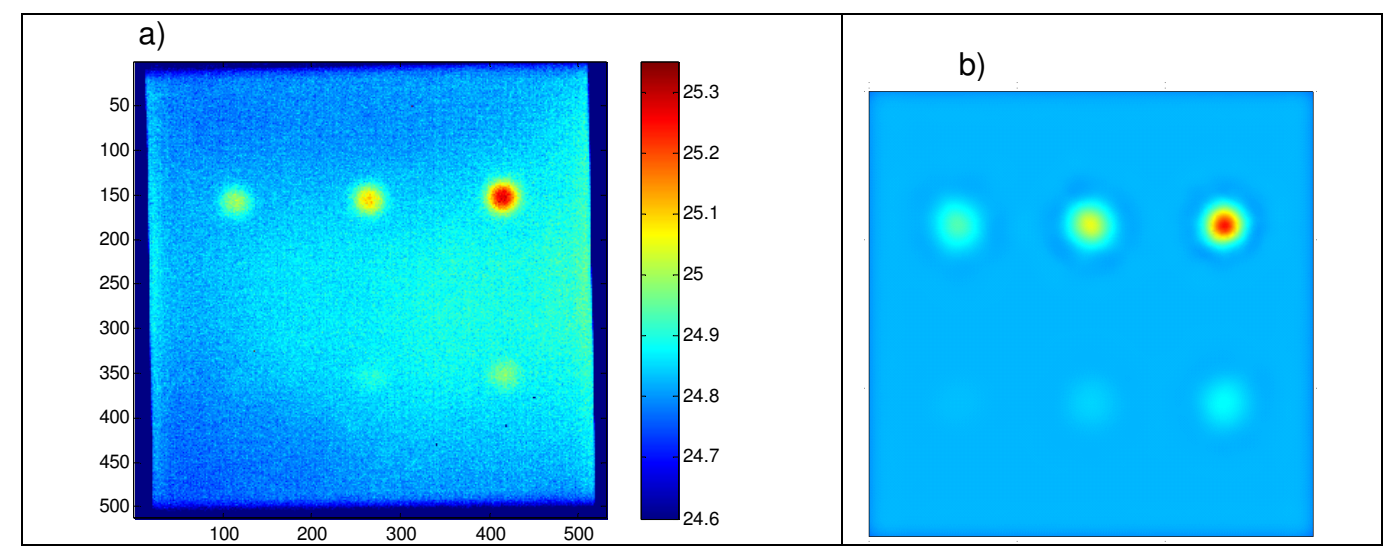

Fig. 3. Flat-bottom hole Plexiglass surface temperature distribution 30 seconds after heat pulse a) experimental results and b) results of numerical model without surface paint layer 
Figure 3a) represents the experimentally obtained thermogram while Figure $3 b$ ) is a corresponding surface temperature solution obtained numerically from a model that had no surface paint. Both temperature scales are adjusted to show the same temperature range in order to enable an easy colour correspondence comparison. Despite the apparent non-uniform heating effects in the experimental results, it can be seen that temperatures of the sane areas correspond relatively well, whereas the defective area temperatures show significant differences.

In order to show the importance of the influence of the surface paint layer, Figure 4 shows the surface temperature decay curves for both cases: the first one where no surface paint layer was included and the second one where a $25 \mu \mathrm{m}$ thick black layer of Paint I was included in the model. Experimentally obtained curves are printed as well, showing that the behavior of the experimental sane zone surface temperature decay curves, which were used as a reference for the determination of the numerical model parameters, correspond to those obtained by simulations. The order of the legend tags presented in the figure corresponds to the order of the curve appearance (top-to-bottom).

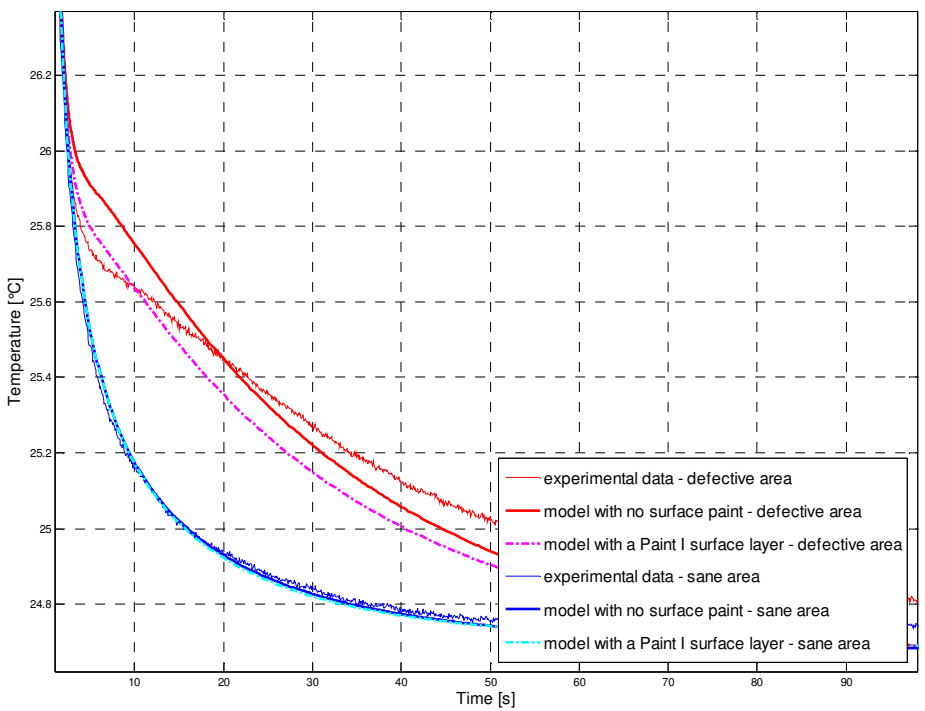

Fig. 4. Surface temperature decay curves: experimental and numerical results for cases with and without $25 \mu \mathrm{m}$ thick black paint layer included in the model

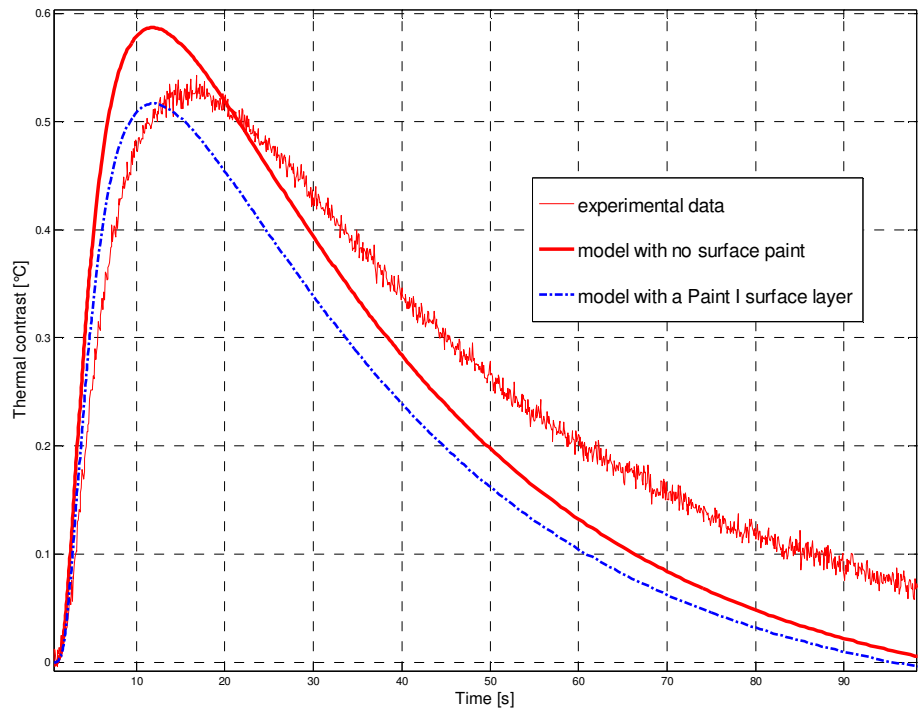

Fig. 5. Thermal contrast: experimental and numerical results for cases with and without $25 \mu \mathrm{m}$ thick black paint layer included in the model

In the case of the shallowest defect located $1 \mathrm{~mm}$ beneath the surface (defect 1 ), the difference is significant and, as shown in Figure 5, this can be seen on the maximum thermal contrast that is reduced to about $82 \%$ of the maximum contrast obtained without a paint layer. The effect is similar for all other defects analyzed.

The results for all six defects are presented in Figure 6. The time of the maximum thermal contrast appearance and the corresponding value of the maximum thermal contrast for each of the 6 defects are shown. The results obtained from models with and without the surface paint layer (Paint I), as well as the experimental 
results are shown. It can be concluded that first the value of the maximum thermal contrast is affected by the surface paint layer, but in addition slight differences can also be noticed on the time of the maximum contrast appearance, more significant for shallow defects than for those further away from the surface.

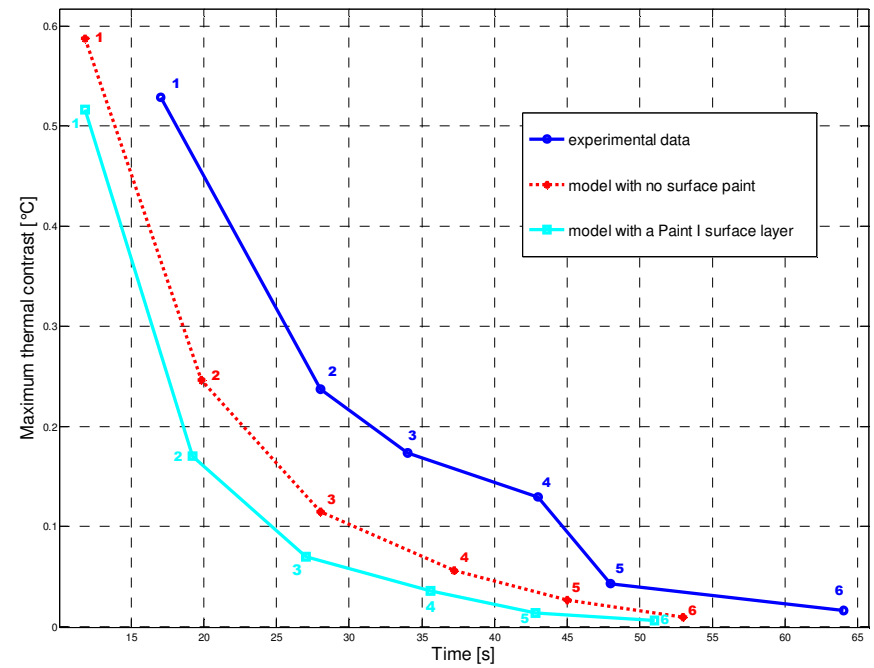

Fig. 6. Maximum thermal contrast and corresponding time of its appearance: comparison between the results of simulations for models with and without surface paint layer (Paint I) and experimental results

In addition, the results of the simulations performed with three surface paints of different thermal properties and of the same thickness were found interesting. The thermal properties of each of the three paints as taken from [16] are listed in Table 1.

Table 1. Thermodynamical properties of paint coatings used for modelling

\begin{tabular}{|l|c|c|c|}
\hline & $\begin{array}{l}\text { Thermal conductivity } \\
{[\mathrm{W} /(\mathrm{mK})]}\end{array}$ & Density $\left[\mathrm{kg} / \mathrm{m}^{3}\right]$ & $\begin{array}{l}\text { Specific heat } \\
\text { capacity }[\mathrm{J} / \mathrm{kgK}]\end{array}$ \\
\hline Paint I & 0.57 & 1162 & 2835 \\
\hline Paint II & 0.74 & 1303 & 2557 \\
\hline Paint III & 1.45 & 1331 & 5184 \\
\hline
\end{tabular}

Figure 7 shows the comparison of the temperature distribution at the time of the maximum contrast achieved for the case of the defect at $2.5 \mathrm{~mm}$ depth from the surface (defect 3), covered with a layer of Paint III. This particular time was chosen because the maximum thermal contrast in the case of the model including the Paint III surface layer showed in general lower values of thermal contrast and therefore, choosing any other time would make the results less illustrative. In the Figure 7, subfigures a), b) and c) correspond to models including 25 $\mu \mathrm{m}$ thick layers of Paint I, Paint II and Paint III respectfully.

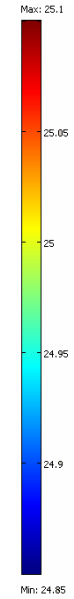

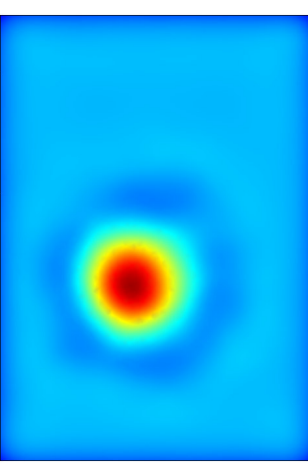

a)

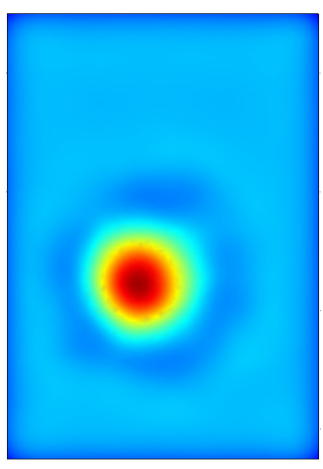

b)

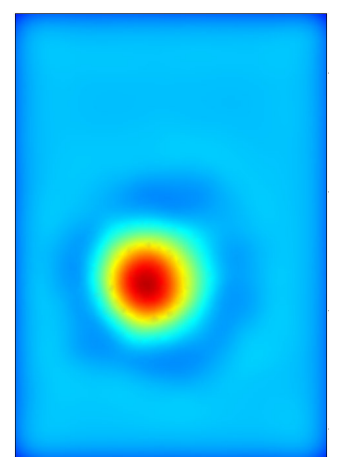

C)

Fig. 7. Surface temperature distribution above the defect at $2.5 \mathrm{~mm}$ depth, simulation results for model with 25 $\mu \mathrm{m}$ thick layer of surface paint coating: a) Paint I, b) Paint II and c) Paint III 
The results of the comparison show that the thermal properties of the paint layer can have an important impact on both the value of the maximum thermal contrast, as well as on the time of its appearance. As seen in Figure 7, the thermal contrast is slightly higher when Paint I and Paint II were used in a model. As was already mentioned, the figures represent the surface temperature distribution at the time when the model with Paint III showed the maximum thermal contrast (21 seconds after heat pulse). That is somewhat later than in case of the first two models, that attained their corresponding maximum thermal contrast about 2 seconds earlier and with a slightly higher value $\left(0.17^{\circ} \mathrm{C}\right.$ compared to $\left.0.15^{\circ} \mathrm{C}\right)$.

Apparently, neither the values of the maximum thermal contrast nor the times of their appearance obtained by simulations are the same as those obtained experimentally. The relative error in maximum thermal contrast for defects at different depths for the model without paint layer, as well as with Paint I surface layer, are presented in Figure 8. For each of the defects the relative error was calculated according to the expression (1).

$$
\varepsilon_{\text {rel }}=\frac{\Delta T_{\text {max }, \exp }-\Delta T_{\text {max }, \text { sim }}}{\Delta T_{\text {max }, \exp }}
$$

From Figure 8 it can be concluded that the model having no paint layer shows variable behavior depending on the defect depth. For the two defects closest to the surface (defect 1 and 2), maximum thermal contrasts obtained numerically are higher than those obtained by experiment, whereas the opposite is true for the deeper defects (defects 3, 4, 5 and 6). On the other hand, the model including a thin surface paint layer shows in all the cases lower values of thermal contrast than the experiment. It can also be observed that, excluding defect 4 which obviously shows a higher value of thermal contrast than one should expect due to the measurement error (see also Figure 6), the relative error is asymptotically approaching a given value (here 0.7 ) and is increasing continuously with the defect depth (the experimental results for the case of the deepest defect should be taken with a lot of reserve since the value of its maximum thermal contrast is of the order of the IR camera sensitivity).

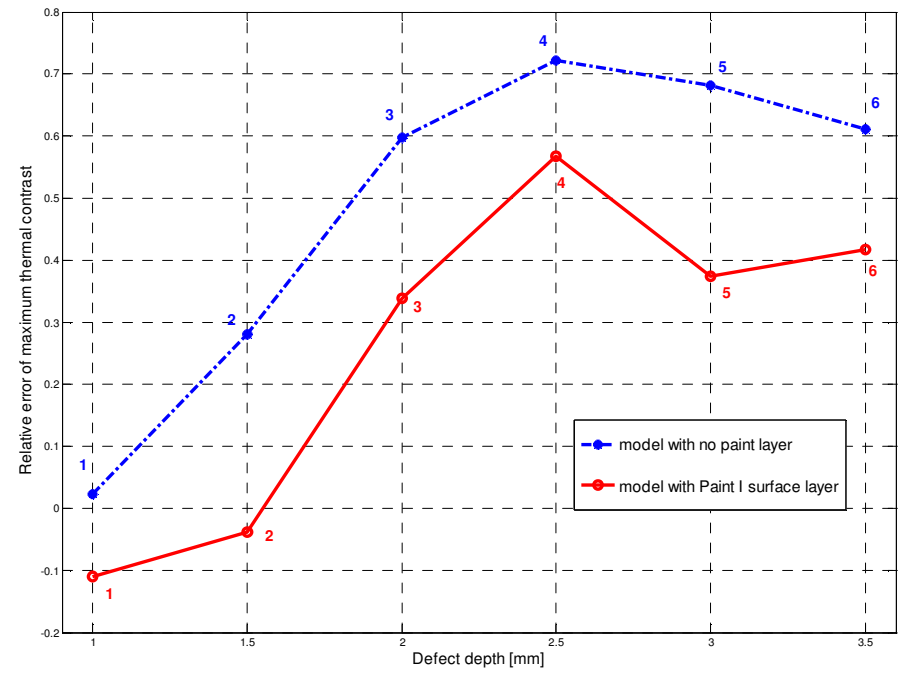

Fig. 8. Comparison of the relative error in maximum thermal contrast for the models with and without a surface paint layer (Paint I)

\section{Conclusion and further work}

The results of the presented analysis imply that characterization procedures based on the maximum thermal contrast should not neglect the impact which surface paints have on the results of simulations. This is particularly important when the maximum thermal contrast values are being considered. The time of the appearance of the maximum thermal contrast also seems to be affected by the existence of the surface paint layer, but to a lesser extent, possibly due to its small thickness. In that sense, physical properties are more important than the actual paint layer thickness. In addition, it has been demonstrated that the relative error in maximum thermal contrast between the experimental and numerical data for the case where the model included the paint layer, has a more natural behavior and, as could be expected, it increases continuously in an asymptotic manner as the defect depth increases.

Further research is directed towards the samples of more conductive materials, such as the metal plate that was previously mentioned in the article. It is expected that large differences in the thermal conductivity of the surface paint layers and the one of the sample material (steel for example), would result in much larger differences in terms of the maximum thermal contrast and the time of its appearance than was the case with the 
Plexiglass sample. However, the speed at which the thermal changes occur in the case of the metal plates subjected to pulse experiment will represent a more important challenge that will have to be dealt with.

\section{Acknowledgements}

Support of NSERC, Canadian Foundation of Innovation and Canada Research Chairs (MiViM) as well as the support of the Ministry of Science, Education and Sports of the Republic of Croatia are acknowledged.

\section{REFERENCES}

[1] Incropera F.P., De Witt D.P.: Fundamentals of heat and mass transfer, John Wiley \& Sons, New York (1990) p 919.

[2] Maldague X.: Theory and practice of infrared technology for nondestructive testing, John Wiley \& Sons, New York (2001).

[3] Delpech P.M., Krapez J.C., Balageas D.L.: Thermal defectometry using the temperature ecay method, Proc. of QIRT 94 (1994) p 220-225

[4] Martin R.E., Gyekenyesi A.L., Shepard S.M.: Interpreting the Results of Pulsed Thermography Data, Materials evaluation (2003) p 611-616.

[5] Almond D.P., Lau S.K.: 'Defect sizing by transient thermography I: an analytical treatment, J. Phys. D: Appl. Phys., Vol 27 (1995) p 2539-2546.

[6] Maillet D. et al., Non-destructive thermal evaluation of delaminations in a laminate: Part I - Identification by measurement of thermal contrast, Composites Science and Technology, Vol 47 (1993) p 137-153.

[7] Maillet D. et al., Non-destructive thermal evaluation of delaminations in a laminate: Part II- The experimental Laplace transforms method', Composites Science and Technology, Vol 47 (1993) p 155-172.

[8] Bendada A. et al.: Interface resistances: The inverse problem for the transient thermal technique, Inverse Problems in Engineering Mechanics, Proc. of the $2^{\text {nd }}$ International Symposium on inverse problems (1994) p 347-354.

[9] Bendada A., Erchiqui F., Lamontagne M.: Pulsed thermography in the evaluation of an aircraft composite using 3D thermal quadrupoles and mathematical perturbations, Inverse problems, Vol 21(2005) p 857-877.

[10] Krapez J.C. et al.:Time-resolved pulsed stimulated infrared thermography applied to carbon-epoxy non destructive evaluation, Proc. of QIRT 92 p195-199

[11] Saintey M.B., Almond D.P.: Defect sizing by transient thermography II: a numerical treatment, J. Phys. D: Appl. Phys., Vol 28 (1995) p 2539-2546.

[12] Krishnapillai M. et al.. Thermography as a tool for damage assessment, Composite Structures, 67 (2005) p 149-155.

[13] Krishnapillai M. et al.: NDTE using pulse thermography: Numerical modeling of composite subsurface defects, Composite Structures, 75 (2006) p 241-249.

[14] Sakagami T., Kubo S., Endo T.: Development of a new processing technique of sequential temperature data after pulse heating for quantitative nondestructive testing, Thermosense XXVI, Proc. of SPIE, Vol. 5405 p 357-365.

[15] Vavilov V.: Un bref panorama des essais non destructifs de structures aérospatiales par méthodes thermiques en URSS, Recherche Aérospatiale, 6 (1991) p 1-16.

[16] Raghu O., Philip J.: Thermal properties of paint coatings on different backings using a scanning photo acoustic technique, Measurement Science and Technology, 17 (2006) p 2945-2949.

[17] Minkowycz W.J., Sparow E.M., Schneider G.E., Plechter R.H.: Handbook of numerical heat transfer, John Wiley \& Sons, New York (1988) p 1024. 
http://dx.doi.org/10.21611/qirt.2008.12_11_16 